\title{
Expression of the candidate fat taste receptors in human fungiform papillae and the association with fat taste function
}

\author{
Dongli Liu ${ }^{1,2}$, Andrew Costanzo ${ }^{1}$, Margaret D. M. Evans ${ }^{3}$, Nicholas S. Archer ${ }^{4}$, Caryl Nowson ${ }^{5}$, \\ Konsta Duesing ${ }^{2 *}$ and Russell Keast ${ }^{1 *}$ \\ ${ }^{1}$ Centre for Advanced Sensory Science, School of Exercise and Nutrition Sciences, Deakin University, Burwood, VIC 3125, Australia \\ ${ }^{2}$ Commonwealth Scientific and Industrial Research Organisation Health E Biosecurity, North Ryde, NSW 2113, Australia \\ ${ }^{3}$ Commonwealth Scientific and Industrial Research Organisation Manufacturing Flagship, North Ryde, NSW 2113, Australia \\ ${ }^{4}$ Commonwealth Scientific and Industrial Research Organisation Agriculture E Food, North Ryde, NSW 2113, Australia \\ ${ }^{5}$ Institute for Physical Activity and Nutrition, Deakin University, Burwood, VIC 3125, Australia
}

(Submitted 26 November 2017 - Final revision received 27 March 2018 - Accepted 7 April 2018)

\begin{abstract}
Significant experimental evidence supports fat as a taste modality; however, the associated peripheral mechanisms are not well established. Several candidate taste receptors have been identified, but their expression pattern and potential functions in human fungiform papillae remain unknown. The aim of this study is to identify the fat taste candidate receptors and ion channels that were expressed in human fungiform taste buds and their association with oral sensory of fatty acids. For the expression analysis, quantitative RT-PCR (qRT-PCR) from RNA extracted from human fungiform papillae samples was used to determine the expression of candidate fatty acid receptors and ion channels. Western blotting analysis was used to confirm the presence of the proteins in fungiform papillae. Immunohistochemistry analysis was used to localise the expressed receptors or ion channels in the taste buds of fungiform papillae. The correlation study was analysed between the expression level of the expressed fat taste receptors or ion channels indicated by qRT-PCR and fat taste threshold, liking of fatty food and fat intake. As a result, qRT-PCR and western blotting indicated that mRNA and protein of CD36, FFAR4, FFAR2, GPR84 and delayed rectifying $\mathrm{K}^{+}$channels are expressed in human fungiform taste buds. The expression level of $C D 36$ was associated with the liking difference score $(R-0.567, \beta=-0.04, P=0.04)$ between high-fat and low-fat food and FFAR2 was associated with total fat intake $(\rho=-0.535, \beta=-0.01$, $P=0.003)$ and saturated fat intake $(\rho=-0.641, \beta=-0.02, P=0.008)$.
\end{abstract}

Key words: Fat taste: Fatty acid metabolism: Fat taste receptors: Delayed rectifying $\mathrm{K}^{+}$channels: RT-PCR: Western blot: Immunohistochemistry

Taste is responsible for the discrimination between foods possessing a positive nutrient composition and substances that may be harmful. Five taste qualities including sweet, umami, bitter, salt and sour tastes provide key information regarding fitness of consumption for a vast array of chemical compounds ${ }^{(1)}$.

The evidence supporting fat as an additional taste is compelling ${ }^{(2-6)}$. There are multiple putative oral peripheral mechanisms associated with fatty acid perception ${ }^{(6)}$, including a number of candidate receptors and ion channels identified to be activated by fatty acids and trigger chemosensory pathway through $\mathrm{Ca}^{2+}$ cascade: CD36 ${ }^{(7,8)}$, FFAR4 (GPR120), FFAR1 $(\text { GPR40 })^{(9,10)}$, FFAR3 (GPR41), FFAR2 (GPR43) $)^{(11)}$, GPR84 $^{(12)}$ and delayed rectifying $\mathrm{K}^{+}$(DRK) channels ${ }^{(13,14)}$. The expression of the candidate receptors and the taste cell types that respond to free fatty acids has not been identified in human gustatory papillae. A comprehensive knowledge of the expression profile of the candidate fat taste receptors is an important step revealing the initial process for fat taste perception at the cellular level.

Taste cells embedded in the taste buds operate as the basic anatomical units for taste function. The taste buds are contained in the tiny bumps on the tongue known as the taste papillae. Three types of gustatory papillae are characterised according to their topographical representation - fungiform, foliate and circumvallate papillae on the anterior, lateral and posterior part of the tongue, respectively ${ }^{(15)}$. In addition, it is reported that there was no significant difference in sensitivity to fatty acids for the three types of the papillae ${ }^{(16)}$, suggesting that all these papillae contain the biological machinery for fat taste detection. The taste cells are classified as four types based on their ultrastructure and function: Type I (basal), Type II (receptor), Type III (presynaptic) and Type IV cells ${ }^{(1)}$.

Peripheral mechanisms for fat taste have been identified, albeit not in humans. CD36 is the best characterised receptor

Abbreviations: DRK, delayed rectifying $\mathrm{K}^{+}$; GPCR, G-protein-coupled receptors; qRT-PCR, quantitative RT-PCR.

* Corresponding authors: Dr K. Duesing, email konsta.duesing@csiro.au; Professor R. Keast, email russell.keast@deakin.edu.au 
among the candidates and is able to detect long-chain fatty acids (LCFA) ${ }^{(17)}$. The evidence for CD36 function as a fat taste receptor includes both $C D 36$ gene knockout in mice ${ }^{(8)}$ and in humans and the association of CD36 SNP rs1761667 with fatty acid taste sensitivity ${ }^{(6,18-21)}$. The G-protein-coupled receptors (GPCR) FFAR4 and FFAR1 are also candidates owing to the ability to bind medium-chain fatty acids (MCFA) and LCFA ${ }^{(9,10)}$, and FFAR3, FFAR2 and GPR84 for SCFA ${ }^{(6,22)}$. However, the absence of FFAR1 in human tongue papillae probably excludes the role of this candidate in human beings ${ }^{(23)}$. DRK channels were reported to respond to cis-PUFA (PUFA) and associated with fatty acid taste sensitivity in rodent models ${ }^{(14,24)}$. Nine types of the DRK channels (KCNA1, KCNA2, KCNA3, KCNA5, KCNA6, KCNB1, KCNB2, KCNC1 and KCNC2) have been well established in Xenopus oocytes or mammalian expression systems, among which KCNA5, KCNC1, KCNC2, KCNA2 and KCNB2 were expressed extensively in anterior rat taste buds ${ }^{(13)}$, with no human study conducted to reveal the expression and function of these channels in human fat taste so far.

Marked individual variations in taste response of the taste system existed across all taste modalities ${ }^{(25,26)}$, which was also observed in fat taste ${ }^{(3)}$. Both genetic and environmental factors were reported to be responsible for the variance. However, data investigating the factors have been controversial. Previous studies suggested that the sensitivity to 6-n-propylthiouracil (PROP), which was largely determined by the genotype, was associated with fat perception ${ }^{(27-29)}$, although this remains controversial $^{(30-32)}$. However, using PROP as a taste function indicator was questioned in many studies based on the lack of correlations between PROP and taste sensitivity to different modalities ${ }^{(31,33-35)}$. Instead, taste papillae density was reported to be partially responsible for taste sensitivity ${ }^{(36-38)}$, with limited studies on fat taste. Recently, variants of the fat taste receptors were associated with oral sensory of fatty acids. The SNP rs1761667 of $C D 36$, which was associated with the expression level of this receptor, was correlated with sensitivity to fatty acids $^{(18,19,21)}$. In addition, some other variants of CD36 and FFAR 4 were related to the taste sensitivity to fatty acids ${ }^{(39-42)}$. It should be noted that some of the SNP such as rs9784998 of CD36 and rs116454156 of FFAR4 were also reported to influence the expression level of the receptors ${ }^{(43,44)}$. This may indicate potential associations between the expression level of the fat taste receptors and fatty acid perception. However, the correlation of fat taste function with the expression level of the fat taste receptors, especially for the GPCR and the DRK channels, remains a gap of our knowledge. For the environmental factor, dietary intake was associated with fat taste sensitivity ${ }^{(45)}$. In addition, other factors such as BMI ${ }^{(3,46-48)}$ and age $^{(49)}$ were reported to be associated with taste sensitivity. As this study included twin participants with a high range of BMI and age, the mixed linear model adjusting for BMI and age, with twin as random effects, was applied to validate the correlations.

Regardless of the level of evidence that exists for each candidate, there is no study to date that has assessed all candidate receptors together and systematically in human taste papillae. This may be partially owing to the lack of reliable antibodies and difficulty in obtaining human taste tissue. The goal of this study was to identify the candidate fat taste receptors that were expressed in human fungiform papillae and their association with fat taste, thus providing the basis for further research to identify the mechanisms associated with fat perception in human oral cavity.

\section{Methods}

\section{Study outline}

This study was conducted according to the guidelines laid down in the Declaration of Helsinki, and all the experimental procedures were approved by the Commonwealth Scientific and Industrial Research Organisation (CSIRO) Human Research Ethics Committee (13/06) and ratified by the Deakin University Human Research Ethics Committee (2014-177). This study contained three cohorts: cohort 1 for gene and protein expression analysis, cohort 2 for immunohistochemistry staining and cohort 3 for association analysis. All participants gave the informed consent before participating in the study and had no history of any taste disorders.

For cohorts 1 and 2, a total of ten participants (six female and four male, aged between 20 and 42 years, with a BMI between 20 and $33 \mathrm{~kg} / \mathrm{m}^{2}$ ) were recruited. For cohort 3, eight pairs of female twins (six monozygotic pairs and two dizygotic pairs, aged between 20 and 62 years, with a BMI between 17 and $35 \mathrm{~kg} / \mathrm{m}^{2}$ ) were included as a subgroup from a larger project recruited by Twins Research Australia.

\section{Fungiform papillae collection}

For participants from all the three cohorts, fungiform papillae biopsy was conducted without anaesthetic by a registered doctor according to the procedure described previously ${ }^{(50,51)}$. The participants fasted overnight before papillae collection. For each participant, six fungiform papillae were collected and pooled as an individual sample. The ten samples collected from cohort 1 and 2 participants were randomly divided into two groups: five samples for RT-PCR analysis and western blotting for cohort 1 and five for immunohistochemistry of cohort 2 .

Cohort 1 and 3 papillae samples were transferred to a vial containing RNALater (Life Technologies) immediately after excision and stored at $-80^{\circ} \mathrm{C}$ before further processing. Cohort 2 papillae tissues were fixed for $3 \mathrm{~h}$ in $4 \%$ neutral buffered formalin (Sigma-Aldrich) and then transferred into $70 \%$ ethanol overnight to preserve antigenic epitopes.

\section{RNA and protein extraction}

To extract RNA and protein, papillae samples of cohorts 1 and 3 were thawed on ice, the RNALater supernatant was taken off and all papillae for each subject were homogenised with a motorised pestle (Sigma-Aldrich) in $1 \mathrm{ml}$ of TRIzol reagent (Life Technologies). The samples were then passed through a 19-gauge needle fifteen times to ensure complete cell lysis. After phase separation with $200 \mu \mathrm{l}$ of chloroform (SigmaAldrich), the RNA was extracted from the aqueous phase according to the manufacturer's protocol ${ }^{(52)}$. The bottom organic layer was retained for protein extraction and was stored at $-80^{\circ} \mathrm{C}$ for later extraction. The RNA pellet was dissolved in 
$20 \mu$ of RNase-free water and treated with DNase to remove contaminating genomic DNA using the RNase-free DNase set (Qiagen). Each sample was then purified overnight by precipitation with $0.3 \mathrm{~m}$ sodium acetate, $2 \mu \mathrm{l}$ of glycogen and 2.5 volumes of $100 \%$ ethanol. After centrifuging and two ethanol washes on the next day, the RNA pellet was re-dissolved in $20 \mu \mathrm{l}$ of RNase-free water. RNA concentrations were measured using the NanoDrop ND-1000 spectrophotometer (Thermo Fisher Scientific) and RNA integrity number (RIN, ranging from 0 to 10 , with 10 indicating the greatest integrity) was assessed using a Bioanalyser 2100 (Agilent Technologies) using the Agilent RNA 6000 Nano Kit. The RNA samples were then stored at $-80^{\circ} \mathrm{C}$ before further processing.

The bottom phenol-ethanol layer of cohort 1 samples was used to extract the protein according to the dialysis protocol of Hummon $^{(53)}$ with minor changes. In short, the samples were loaded in dialysis membranes (MWCO 6000; Spectra/Por) against three changes of $0 \cdot 1 \%$ sodium dodecyl sulfate (SDS) at $4^{\circ} \mathrm{C}$ for 16,4 and $2 \mathrm{~h}$ continuously. The resultant mass was dissolved in $200 \mu \mathrm{l}$ of SDS sample buffer $(62.5 \mathrm{~mm}$ TRIS-HCl, $1 \mathrm{~mm}$ dithiothreitol, $2 \%$ SDS, $10 \%$ glycerol) containing protease inhibitor cocktail (Roche) at $50^{\circ} \mathrm{C}$ in a water bath for $15 \mathrm{~min}$. The protein concentrations were estimated using the Pierce ${ }^{\mathrm{TM}}$ BCA protein assay kit (Life Technologies) and stored at $-20^{\circ} \mathrm{C}$.

\section{Phenotyping tests}

The participants for cohort 3 were asked to participate in a 2-h laboratory session one day before the papillae collection. The session was conducted in a temperature- and sound-controlled partitioned sensory booth consisting of four sub-sessions: (1) anthropometry measurement, which includes the body weight and height measurement without shoes and in light clothing; (2) three-pass 24-hour dietary recall ${ }^{(54)}$ conducted by a trained nutritionist; (3) detection threshold to oleic acid (C18:1) measurement with a duplicate ascending series 3-alternate force choice (3-AFC) methodology ${ }^{(55)}$; and (4) liking rating analysis for high-fat and low-fat foods based on seven pairs of high-fat foods and their reduced-fat counterparts. For the detection threshold and liking rating analysis, the samples were prepared according to the same procedure as detailed by ${ }^{(56)}$. After the sub-session 1, BMI was calculated as weight $(\mathrm{kg}) /$ height $(\mathrm{m})^{2}$. The amount of energy intake $(\mathrm{kJ})$, fat intake (in the unit of $\mathrm{g}$, including total, saturated, polyunsaturated and monounsaturated fat intake) and the percentage of the energy derived from fat (including total, saturated, polyunsaturated and monounsaturated fat percentage) were calculated based on the data from the 24-h dietary recall using computer software FoodWorks (version 8; Xyris). Mean fat taste detection threshold (mM) was determined for each participant based on duplicate 3-AFC sessions, and transformed to an ordinal scale labelled as fat taste rank (ranging from 0 to 12, with higher ranks implying higher detection threshold) ${ }^{(56)}$. The liking scores of high fat and low fat rated from -100 to 100 were calculated based on the mean scores of the seven high-fat or low-fat foods. The liking difference scores were calculated based on the mean liking score of the high-fat foods minus that of the low-fat foods. The higher liking difference score indicated a higher level of the preference to high-fat food compared with low-fat food, and vice versa. The relative liking score was used in the following correlation analysis to eliminate the individual bias in the scores.

\section{Quantitative RT-PCR analysis}

The gene expression of all candidate fat taste receptors including CD36, the GPCR and the nine DRK channels was analysed with quantitative RT-PCR (qRT-PCR) initially. For the RNA samples extracted from cohort 1 and 3 samples, $1 \mu \mathrm{g}$ of total RNA was used to synthesise complementary DNA (cDNA) using the High Capacity cDNA Reverse Transcription Kit (Applied Biosystems) and each cDNA sample was diluted 1:5 with RNase-free water. Standards were prepared with a serial dilution of 1:5 from the top standard (an aliquot of the all the 1:5 dilution cDNA samples). The gene expression of all candidate fat taste receptors including CD36, the GPCR and the DRK channels expressed extensively in Liu et al. ${ }^{(13)}$ were quantified for cohort 1 samples using the Taqman gene expression assays as detailed in Table 1. Only the positively expressed genes were analysed with the corresponding Taqman gene expression assays (listed in Table 1) for cohort 3 samples. Four replicates of each cDNA sample were analysed in a 384-well plate format with the Lightcycler 480 Real-time PCR Instrument (Roche) and run for forty cycles of the PCR programme. For each gene analysis, a negative control of the sample that had not been reverse-transcribed and a positive control from RNA isolated from whole blood were included. GAPDH and RPLPO were included as reference genes for normalising the transcript numbers. All genes analysed were found to be expressed in human whole blood according to the gene expression omnibus (GEO) profiles of National Centre for Biotechnology Information (https://www.ncbi.nlm.nih.gov/geoprofiles).

\section{Western blotting analysis}

The genes that failed to be amplified in all of the five samples during the forty cycles from the last step were not proceeded to protein expression analysis. For the protein extracted from cohort 1 samples, same amount $(20 \mu \mathrm{g})$ of each sample was

Table 1. Taqman expression assays for the quantitative RT-PCR analysis

\begin{tabular}{lll}
\hline Genes & Assay ID & Description \\
\hline CD36 & Hs01567185_m1 & Probe spans exons \\
FFAR4 & Hs00699184_m1 & Probe spans exons \\
FFAR1 & Hs03045166_s1 & Probes are within a single exon \\
FFAR3 & Hs02519193_g1 & Probes are within a single exon \\
FFAR2 & Hs00271142_s1 & Probes are within a single exon \\
GPR84 & Hs01874713_s1 & Probes are within a single exon \\
KCNA1 & Hs00264798_s1 & Probes are within a single exon \\
KCNA2 & Hs04187587_g1 & Probe spans exons \\
KCNA3 & Hs04403047_m1 & Probe spans exons \\
KCNA5 & Hs00969279_s1 & Probes are within a single exon \\
KCNA6 & Hs00266903_s1 & Probes are within a single exon \\
KCNB1 & Hs00270657_m1 & Probe spans exons \\
KCNB2 & Hs00191116_m1 & Probe spans exons \\
$K C N C 1$ & Hs00428197_m1 & Probe spans exons \\
KCNC2 & Hs01066923_m1 & Probe spans exons \\
$R P L P O$ & Hs99999902_m1 & Probe spans exons \\
GAPDH & Hs02758991_m1 & Probe spans exons \\
\hline
\end{tabular}


Fat taste receptors in human fungiform papillae

Table 2. Primary and secondary antibodies used for western blotting analysis

\begin{tabular}{lllll}
\hline Primary antibodies & Source & Dilution & Catalogue no. & Secondary antibody* \\
\hline Anti-CD36 & Santa Cruz & $1: 500$ & sc7309 & Goat anti-mouse IgG-HRP \\
Anti-FFAR4 & Abcam & $1: 1000$ & ab118757 & Goat anti-rabbit IgG-HRP \\
Anti-FFAR2 & Sigma-Aldrich & $1: 500$ & SAB4501283 & Goat anti-rabbit IgG-HRP \\
Anti-GPR84 & Santa Cruz & $1: 1000$ & sc99106 & Goat anti-rabbit IgG-HRP \\
Anti-KCNA2 & Abcam & $1: 1000$ & ab65789 & Goat anti-rabbit IgG-HRP \\
\hline
\end{tabular}

* The secondary antibodies are all from Bio-Rad, used with a dilution of 1:3000.

boiled for 5 min in Pierce ${ }^{\mathrm{TM}}$ Lane Marker Reducing Sample Buffer (Life Technologies). All of the samples were then separated on $10 \%$ Mini-PROTEAN $^{\circledR}$ TGX ${ }^{\mathrm{TM}}$ Gel (Bio-Rad) and transferred onto the PVDF membranes (Bio-Rad). The membranes were then blocked with $5 \%$ non-fat milk in TRIS Buffered Saline containing $0 \cdot 1 \%$ Tween-20 (TBST) for $1 \mathrm{~h}$ at room temperature before being incubated with the primary antibody (Table 2) at $4^{\circ} \mathrm{C}$ overnight. The specificity of the antibodies was validated with specific positive controls and negative controls (shown in the online Supplementary data). On the next day, all the membranes including the one incubated without the primary antibody were washed three times with $0 \cdot 1 \%$ TBST (10 min each) and then incubated with the corresponding conjugated secondary antibody (detailed in Table 2) for $1 \mathrm{~h}$ at room temperature. The final washes were conducted after incubation with $0.1 \%$ TBST five times ( 5 min each). The immunoreactivity was then visualised using the ECL system (Bio-Rad).

\section{Immunohistochemistry localisation}

For cohort 2 papillae samples, individual papilla were oriented in a micro-well filled with $2 \%$ agarose before embedding and sectioning to ensure that the papilla was sectioned in the longitudinal way. The solidified agarose containing papillae was then processed through the normal dehydration and paraffin infiltration procedure using a Tissue Processor (Leica TP1050), and was reorientated before embedding in paraffin. The 5- $\mu$ m-thin serial sections were cut with a RM2235 Rotary microtome (Leica Microsystems), mounted on slides, air-dried and stored in cutting order in an airtight box at room temperature for immunostaining. To ensure the orientation of the section (longitudinal sections) and the detection of the possible taste bud structures, approximately every 5 th section was routinely stained with haematoxylin-eosin (H\&E), mounted and viewed using routine light microscopy. Once the taste buds were detected from the H\&E staining, the neighbouring slides were chosen for the following immunohistochemistry staining

The candidate receptors and ion channels were stained using either immunofluorescence (FFAR4, GPR84 and KCNA2) or immunoperoxidase labelling (CD36 and FFAR2) based on the results of a pilot antibody optimisation study. For immunofluorescence staining, the paraffin sections were first deparaffinised with three xylene washes and rehydrated in a descending series of alcohol using 100, 75 and 50\% ethanol. Sections were then treated with $0 \cdot 1 \mathrm{~m}$ sodium citrate buffer $(\mathrm{pH}$ 6.0) for $2 \mathrm{~min}$ in a microwave at $1000 \mathrm{~W}$ for antigen retrieval. After being rinsed with PBS, the slides were blocked with
Image-iT FX signal enhancer (I36933; Life Technologies) for $30 \mathrm{~min}$, followed by incubation in $10 \%$ normal donkey serum (NDS) in PBST $(0 \cdot 3 \%$ Triton X100 in PBS) with $1 \%$ bovine serum albumin (BSA) for $1 \mathrm{~h}$ at room temperature. The sections were incubated with anti-PLC $\beta 2$ (sc-31757; Santa Cruz Biotechnology) diluted 1 part in 50 with $2 \%$ NDS overnight at $4^{\circ} \mathrm{C}$ and thoroughly rinsed with PBS. Finally, the sections were incubated with the donkey anti-goat $\operatorname{IgG}(\mathrm{H}+\mathrm{L})$ conjugated with Alexa 488 (A-150129; Life Technologies), 1:500 for $1 \mathrm{~h}$ at room temperature and mounted in Fluoroshield with DAPI (Sigma-Aldrich) and viewed using an inverted fluorescence microscope (Nikon) with image acquisition controlled by the NIS Elements AR 4.30.00 software (Nikon).

For immunoperoxidase labelling, sections were deparaffinised and rehydrated, and the endogenous alkaline phosphatase and peroxidase activity was inhibited with BLOXALL blocking solution (Vector Laboratories), followed by the antigen retrieval process described previously. After being rinsed in PBS, the sections were blocked for 30 min with $5 \%$ BSA in PBST at room temperature. The primary antibodies (detailed in Table 3) were diluted in Da Vinci green antibody diluent (Biocare Medical) at a concentration ranging from 5 to $20 \mu \mathrm{g} / \mathrm{ml}$ or neat universal control Ig overnight at $4^{\circ} \mathrm{C}$. After several PBS washes, the sections were incubated with either goat antimouse or goat anti-rabbit biotinylated secondary antibody (Zymed Laboratories) for $30 \mathrm{~min}$ at $37^{\circ} \mathrm{C}$. Next, the sections were rinsed and incubated with streptavidin-HRP (streptavidinhorseradish peroxidase; Vector Laboratories) for $30 \mathrm{~min}$. After the unbound streptavidin-HRP was removed by thoroughly rinsing with PBS, the DAB system (Vector Laboratories) was applied for signal detection. The sections were counterstained with haematoxylin and mounted after the final wash. Stained sections were viewed using bright-field microscopy on the same inverted microscope (Nikon) with the same acquisition system. For both staining methods, a negative control (isotype control) of the Ig from the same species of the animal (gift from Dr M. D. M. E. from CSIRO Agriculture \& Food) as the primary antibody was run simultaneously with all the sections in order to verify the positive signals of the result. All of the images were captured at the same exposure time for specific magnification.

\section{Statistics}

The expression level of a target gene was calculated using the relative quantification of the target gene against two reference genes (arithmetic mean of RPLPO and GAPDH transcripts). Standard curves were generated to determine the amplification efficiency $\left(10^{-1 / \mathrm{m}}\right.$, where $\mathrm{m}$ is the gradient of the standard 
Table 3. Primary antibodies used in the immunohistochemistry analysis

\begin{tabular}{lccll}
\hline Primary antibodies & Source & Dilution & Catalogue no. & Secondary antibody \\
\hline Anti-PLCB2 & Santa Cruz & $1: 50$ & sc31757 & Donkey anti-goat IgG-Alexa Fluor 488* \\
Anti-CD36 & Santa Cruz & $1: 50$ & sc7309 & Biotinylated goat anti-mouse IgG \\
Anti-FFAR4 & Novus Biologicals & $1: 500$ & NBP1-00858 & Donkey anti-rabbit IgG-Alexa Fluor 488* \\
Anti-FFAR2 & Abnova & $1: 300$ & PAB16402 & Biotinylated goat anti-rabbit IgG \\
Anti-GPR84 & Santa Cruz & $1: 300$ & sc99106 & Donkey anti-rabbit IgG-Alexa Fluor 488* \\
Anti-KCNA2 & Abcam & $1: 50$ & ab65789 & Donkey anti-rabbit IgG-Alexa Fluor 488* \\
\hline
\end{tabular}

* The secondary antibodies are from Life Technologies, used with a dilution of 1:500.

$\dagger$ The secondary antibodies are from Zymed Laboratories, used with a dilution of 1:50.

curve) of the PCR reaction. The minimally detectable effect size $|\rho|$ was 0.64 determined by Gpower (version 3.1.9.2) with $\alpha$ (probability of type I error) of $0 \cdot 05,80 \%$ power and the sample size of 16 (two tails). The statistical analysis for the correlation study was conducted using SPSS package (version 24.0). At first, the normality of the gene expression level in terms of the relative transcript numbers resulted from qRT-PCR and the phenotyping data were assessed in the Shapiro-Wilk test to indicate whether they followed the normal distribution. The correlation analysis was first estimated with either Pearson's moment correlation coefficient $(R)$ for normal distributed pairs of interested variances or Spearman's rank correlation coefficient $(\rho)$ for the other pairs. Then linear mixed-effects models were conducted to assess the associations between pairs of interested variables including age and BMI as fixed effects and the twin pair as a random effect to validate the associations. Age and BMI were included as covariates to adjust for the effect on taste. The statistical significance was set at $P<0.05$.

\section{Results}

\section{Gene expression of the candidate fat taste receptor genes}

High-quality RNA was extracted from biopsied fungiform papillae with Bioanalyser RIN values between 7.5 and 9.2. Gene expression was observed for CD36, FFAR4, FFAR2, GPR84 and $K C N A 2$ in all of the fungiform papillae samples. Fig. 1 shows the normalised transcripts level of each gene in the five papillae samples. Considerable amplification of the genes CD36, FFAR4, FFAR2, GPR84 and KCNA2 was observed in all of the samples after forty PCR cycles. No gene expression was observed for FFAR1, FFAR3, KCNA5 and KCNC1 in any of the samples, suggesting that these are unlikely to function as candidate fat taste receptors in human fungiform papillae. KCNB2 and KCNC2 showed low levels of amplification, including no amplification in some of the samples. All Taqman assays resulted in amplification of positive controls (RNA samples from whole blood) and no amplification of negative controls (samples without being reverse-transcribed).

\section{Detection of the candidate fat taste receptor proteins}

Western blot analysis was completed to confirm expression for the positively expressed genes identified by the qRT-PCR in the same fungiform papillae samples. All of the candidate receptor and DRK channel proteins were detected from the human fungiform papillae protein lysates (Fig. 2).

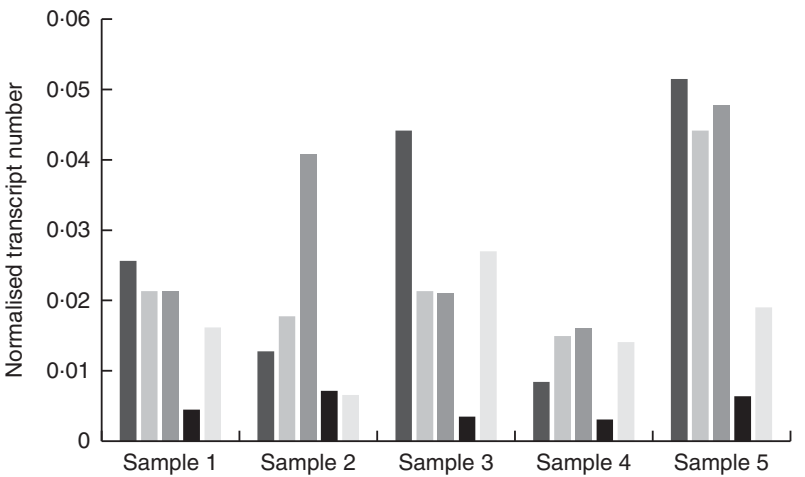

Fig. 1. Quantitative RT-PCR results of the candidate fat taste receptors and delayed rectifying $\mathrm{K}^{+}$channels in human fungiform papillae. The relative transcript numbers represent transcript numbers normalised against the averaged transcript number of the housekeeping genes (RPLPO and GAPDH). $\square$, CD36; $\square$, FFAR4; $\square$, FFAR2; $\square$, GPR84; $\square$, KCNA2.
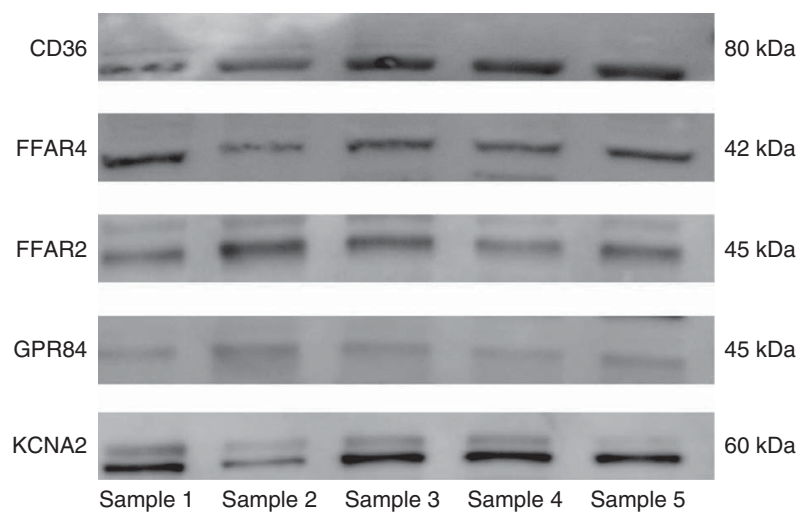

Fig. 2. Western blot results of the candidate fat taste receptor and delayed rectifying $\mathrm{K}^{+}$channel proteins having positive gene expression from human fungiform papillae.

\section{Localisation of the fat taste receptors in human fungiform taste buds}

The candidate fat taste receptors displaying positive gene and protein expression profiles in human fungiform papillae were next assessed using immunohistochemistry analysis to identify the specific cell/tissue type origin (i.e. was expression originating from the taste bud, or another cell type). H\&E staining of papillae sections revealed that approximately $20 \%$ sections contained obvious taste bud structures (Fig. 3(a), dashed circles).

The immunoenzymatic staining results (Fig. 3) show that the positively expressed candidates that resulted from western blots 

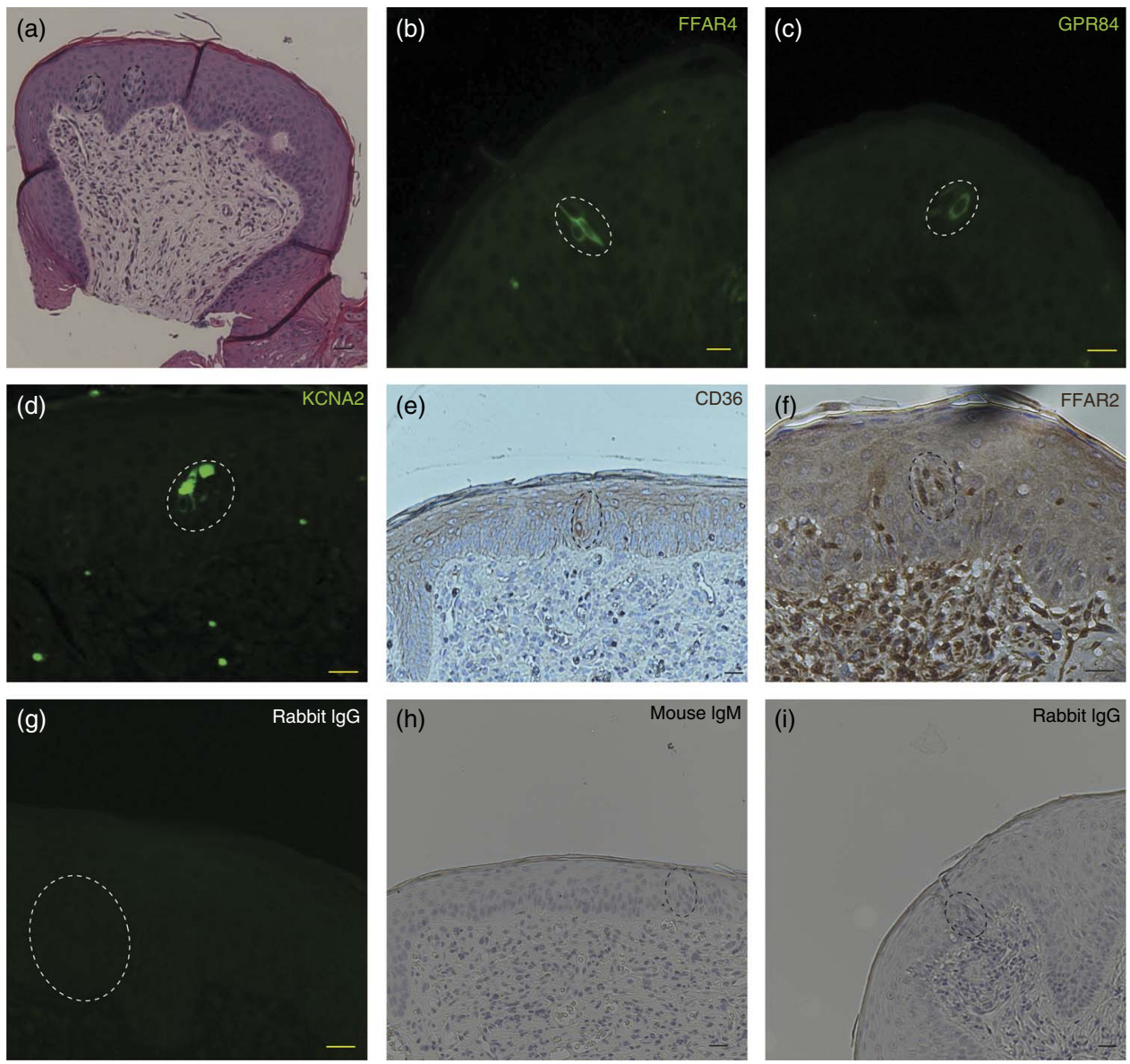

Fig. 3. Representative images of positive taste bud structures in fungiform papillae sections stained with (a) haematoxylin-eosin (H\&E) and the selected panel of antibodies (b) FFAR4, (c) GPR84, (d) KCNA2, (e) CD36 and (f) FFAR2 and (g-i) negative controls, performed using either immunoperoxidase (e, f, h, i) or fluorescentconjugated secondary antibodies (b, c, d, g). The dashed circles denote identifiable taste bud structures in each section. Scale bars represent $50 \mu \mathrm{m}$ in the H\&Estained section (a), fluorescent images (b, c, d, g) and in immunoperoxidase images (e, f, h, i).

were present in fungiform taste buds. The taste bud structures (highlighted with dashed circles in Fig. 3) were identified alongside the corresponding light image from the same panel where the fluorescence figures were taken. The positive signals were verified when they were absent from the corresponding negative control slides (Fig. 3(g)-(i)). Receptors for LCFA (CD36 and FFAR4), MCFA (GPR84), SCFA (FFAR2) and cis-PUFA (KCNA2) were identified in human fungiform papillae, in an outcome that was consistent with the expression data from the western blotting technique (as presented in Fig. 2). All of the candidate receptors were also located in taste buds, although some of the staining was faint in the taste tissue (for GPR84 and FFAR2). Besides the taste buds, the receptors CD36, FFAR2 and the DRK channels had positive expression present in other tissue types in the fungiform papillae. The receptors FFAR4 and GPR84 showed expression specifically in taste cells only. From the gene, protein expression and immunohistochemistry localisation studies, CD36, FFAR4, FFAR2, GPR84 and KCNA2 were analysed with the following correlation study.

\section{Correlation of the fat taste receptors and fat taste phenotypes}

The distribution of the relative expression levels of $C D 36$ and KCNA2 from qRT-PCR and all the phenotyping factors was normal ( $P>0.05$ from Shapiro-Wilk test), whereas the expression levels for FFAR2, FFAR4 and GPR84 were not. Therefore, the correlation coefficient for the expression level of $C D 36$ and KCNA2 and the fat taste function indicators was estimated with the Pearson's correlation analysis, whereas the other genes were analysed with Spearman's $\rho$. The correlation coefficients and the $P$ values are shown in Table 4 . From the analysis, the expression level of $C D 36$ presented a negative correlation with the preference to high-fat foods (indicated by the liking difference score) and a positive association with total fat, saturated fat intake and the energy from saturated fat proportion in $24 \mathrm{~h}$. In addition, the expression level of FFAR2 was negatively associated with energy intake, saturated fat intake and monounsaturated fat intake for $24 \mathrm{~h}$. No correlations of the detection threshold to oleic acid or unsaturated fat intake was observed for any of the taste receptor genes. The significant correlations were validated in the mixed model regression analysis, taking twin pair as a random effect and adjusted for age and BMI. After taking the twin pair, age and BMI into consideration, the significant association was observed for expression level of $C D 36$ with the liking difference score $(\beta=-0 \cdot 04, P=0 \cdot 04)$, FFAR2 with total fat intake $(\beta=-0.01, P=0.003)$ and saturated fat intake $(\beta=-0.02, P=0.008)$ for $24 \mathrm{~h}$. The other significant 
D. Liu et al.

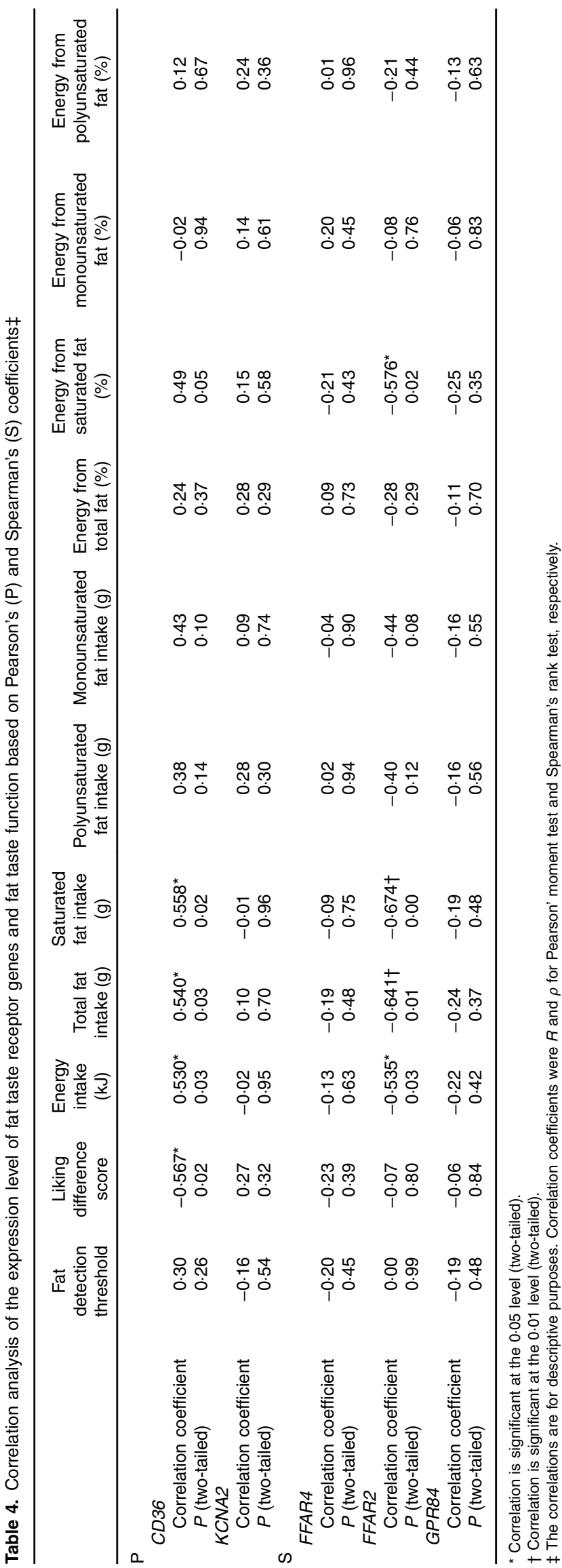

correlations were not observed with twin pair as random effect and age and BMI as fixed effects.

\section{Discussion}

This study is the first to systematically examine putative candidate receptors responsible for fat taste in human fungiform papillae. Previous studies identified $\mathrm{CD} 36$ in human tongue papillae $e^{(57,58)}$ and FFAR4 in human taste tissue of fungiform papillae ${ }^{(58)}$ and circumvallate papillae ${ }^{(23)}$. From the gene and protein expression analysis result in our study, CD36, FFAR4, FFAR2, GPR84 and certain types of DRK channels, such as KCNA2, are present in human fungiform papillae samples. The quantitative analysis of the gene expression levels further indicated significant correlations among many of the fat receptor genes. The immunostaining serves to confirm the expression of their protein products, further underscoring the likely role they may play in identification of the fatty acids in foods. These candidate receptors, as well as some of the others not seen in fungiform papillae, may be present in other types of gustatory papillae in the human oral cavity. Therefore, the absence of receptors in fungiform papillae does not exclude their role in fat taste perception in human. It should also be noted that candidate fat taste receptors are present broadly in other tissues and performed different roles. For example, CD36 expression has been identified in blood and involved with cell adhesion $^{(59)}$ and digestive secretion ${ }^{(60)}$.

Our study shows a divergent expression profile of the candidate fat taste receptors in humans compared with rodent models. For example, KCNA5, serving as the major DRK channel expressed in rat tongue ${ }^{(13)}$, was not identified in the human fungiform papillae. In contrast, KCNA2 appears to be the most abundant DRK channel in human fungiform papillae. The expression analysis revealed by qRT-PCR also indicates that the predominant sub-family of the DRK channels in human fungiform papillae appears to be the Shaker channels (KCNA) rather than the Shab (KCNB) or Shaw (KCNC) channels, which is consistent with rodent models ${ }^{(13)}$. The candidate DRK channels selected were based on the DRK expression analysis from rat $^{(13)}$, but as there appears divergent expression profiles between rat and human, there might be other DRK channels expressed in human that do not overlap with those identified in rodent models. Future studies are required to explore the expression profile of further DRK channel types in relation to their function in human fat taste.

Another main distinction is the absence of FFAR1 and FFAR3 in human fungiform papillae. FFAR1 has been reported to expand the types of fat stimuli with its affinity to shorter MCFA $^{(61)}$ and was found to be expressed in mice circumvallate papillae ${ }^{(62)}$. However, FFAR1 was not expressed in human fungiform papillae in a previous study ${ }^{(23)}$, which was consistent with what was found in this study. Although FFAR1 was absent in human fungiform papillae, the existence of GPR84 in taste buds of the tongue papillae fills in the gap of the detection of certain MCFA, as some of the agonists overlap between FFAR1 and GPR84 ${ }^{(12)}$. In addition, the expression of GPR84 in granulocytes suggests a potential role it might play in the immune system of the oral cavity ${ }^{(63)}$. 
FFAR3 and FFAR2 were always reported by previous studies simultaneously as they belong to a sub-family of homologous GPCR. They are both tuned to SCFA such as acetic acid, and trigger the similar downstream signal transduction pathway, albeit through different mediators ${ }^{(11)}$. Although controversies arose when it was shown that the taste perception of SCFA overlaps largely with sour taste ${ }^{(5)}$, the signal transduction mechanisms for SCFA and common sour stimuli showed inconsistency. Data from previous studies attempting to validate the candidate sour taste receptor-polycystic kidney disease (PKD) channels showed that acetic acids triggers a response of non-transfected human HEK293T cells (highly transfectable derivative of human embryonic kidney 293 cells), as well as PKD1L3- and/or PDK2L1-transfected HEK293T cells, which was not identified for other acid solutions tested ${ }^{(64,65)}$. The expression of FFAR3 and FFAR2 in kidney cells ${ }^{(66)}$ might explain the increase in intracellular calcium ion concentration evoked by acetic acids independent from the PKD channel pathway. In the current study, FFAR2 was identified in human fungiform papillae, including the taste bud tissue, whereas FFAR3 was absent. This was not unexpected as the expression of the two receptors is reported to be highly tissue specific: FFAR2 is reported to be expressed predominantly in immune cells such as neutrophils and monocytes ${ }^{(67,68)}$ and FFAR3 mainly in blood mononuclear cells and adipose tissue ${ }^{(11,69)}$.

Most of previous studies associated the oral sensory perception of fatty acids with CD36, FFAR4 and FFAR1. A major finding from the association analysis is that CD36 showed a significant negative association with the difference in liking between high-fat and low-fat foods. As obese individuals showed a preference for diets higher in fat content ${ }^{(70)}, \mathrm{CD} 36$ may play a role in the development of obesity through regulating fat sensing. Previous studies showed a negative correlation between the detection threshold to C18:1 and the expression level of $\mathrm{CD}_{3} 6^{(18)}$. However, none of the genes showed a significant correlation with the detection threshold to C18: 1 in this study, which could be owing to the small sample size or large variability between populations. As this study is part of a large project, the sample size is underpowered to detect all associations. Besides, genetic variants on chemosensory receptors across populations were reported to be associated with taste perception and food habits ${ }^{(71)}$. The variants that were not associated with the expression of the fat taste receptors could also play a role in fat taste functions and introduce large deviations in the association analysis. The genotypes of the taste receptor genes were not analysed in this study, but could also have yielded insights into the association analysis. Our association analysis also showed that FFAR2 expressed in human fungiform papillae was related to the fat intake. Future functional analysis is required to validate the roles these fat taste receptors play in oral fat perception.

The presence of multiple receptors and mechanisms responsive to fatty acids on taste cells implies that a sophisticated system may underpin fat taste perception. A crosstalk model that combines multiple receptors and ion channels has been previously proposed ${ }^{(72,73)}$, although this is controversial $^{(6)}$. Here we have identified several receptors in taste tissue that possess the ability to bind fatty acids, which suggests a crosstalk model or, alternatively, different receptors tuned to different fatty acid lengths/types may also be possible. By assessing systematically the expression profile of all candidate fatty acid taste receptors reported thus far, this work provides a platform for further research to identify the receptor(s) and mechanism(s) for the detection of fatty acids in humans. Future co-localisation studies are required to identify taste cell sub-type and co-expression patterns of the candidate receptors in human taste tissue. Additionally, the response of the candidate receptors to dietary intervention would assist in further elucidating the mechanism(s) of fatty acid taste detection.

\section{Acknowledgements}

The authors express gratitude to the study volunteers who donated samples.

This work was funded by the National Health and Medical Research Council (NH\&MRC) grant 104780.

The study was designed by D. L., N. S. A., K. D. and R. K. D. L. conducted the experiments, analysed the data and wrote the paper. A. C. contributed to the phenotyping tests. M. D. M. E., N. S. A. and K. D. contributed to the development of the methodology and provided technical assistance. R. K., K. D. and C. N. contributed to the acquisition of the funding and supervision of the project. All authors reviewed the results and approved the final version of the manuscript.

The authors declare that there are no conflicts of interest.

\section{Supplementary material}

For supplementary material/s referred to in this article, please visit https://doi.org/10.1017/S0007114518001265

\section{References}

1. Chaudhari N \& Roper SD (2010) The cell biology of taste. J Cell Biol 190, 285-296.

2. Chalé-Rush A, Burgess JR \& Mattes RD (2007) Evidence for human orosensory (taste?) sensitivity to free fatty acids. Chem Senses 32, 423-431.

3. Stewart JE, Feinle-Bisset C, Golding M, et al. (2010) Oral sensitivity to fatty acids, food consumption and BMI in human subjects. Br J Nutr 104, 145-152

4. Keast RS \& Costanzo A (2015) Is fat the sixth taste primary? Evidence and implications. Flavour $\mathbf{4}, 1$.

5. Running CA, Craig BA \& Mattes RD (2015) Oleogustus: the unique taste of fat. Chem Senses $\mathbf{4 0}, 507-516$.

6. Liu D, Archer N, Duesing K, et al. (2016) Mechanism of fat taste perception: association with diet and obesity. Prog Lipid Res 63, 41-49.

7. Baillie A, Coburn C \& Abumrad N (1996) Reversible binding of long-chain fatty acids to purified FAT, the adipose CD36 homolog. J Membr Biol 153, 75-81.

8. Laugerette F, Passilly-Degrace P, Patris B, et al. (2005) CD36 involvement in orosensory detection of dietary lipids, spontaneous fat preference, and digestive secretions. J Clin Invest 115, 3177-3184.

9. Briscoe CP, Tadayyon M, Andrews JL, et al. (2003) The orphan $\mathrm{G}$ protein-coupled receptor GPR40 is activated by medium and long chain fatty acids. J Biol Chem 278, 11303-11311. 
10. Kotarsky K, Nilsson NE, Flodgren E, et al. (2003) A human cell surface receptor activated by free fatty acids and thiazolidinedione drugs. Biochem Biophys Res Commun 301, 406-410.

11. Brown AJ, Goldsworthy SM, Barnes AA, et al. (2003) The orphan G protein-coupled receptors GPR41 and GPR43 are activated by propionate and other short chain carboxylic acids. J Biol Chem 278, 11312-11319.

12. Wang J, Wu X, Simonavicius N, et al. (2006) Medium-chain fatty acids as ligands for orphan $G$ protein-coupled receptor GPR84. J Biol Chem 281, 34457-34464.

13. Liu L, Hansen DR, Kim I, et al. (2005) Expression and characterization of delayed rectifying $\mathrm{K}^{+}$channels in anterior rat taste buds. Am J Physiol Cell Physiol 289, C868-C880.

14. Gilbertson TA, Liu L, York DA, et al. (1998) Dietary fat preferences are inversely correlated with peripheral gustatory fatty acid sensitivity. Ann N Y Acad Sci 855, 165-168.

15. Chandrashekar J, Hoon MA, Ryba NJP, et al. (2006) The receptors and cells for mammalian taste. Nature $\mathbf{4 4 4}$, 288-294.

16. Mattes RD (2009) Oral thresholds and suprathreshold intensity ratings for free fatty acids on 3 tongue sites in humans: implications for transduction mechanisms. Chem Senses 34, 415-423.

17. Febbraio M, Hajjar DP \& Silverstein RL (2001) CD36: a class B scavenger receptor involved in angiogenesis, atherosclerosis, inflammation, and lipid metabolism. J Clin Invest 108, 785-791.

18. Pepino MY, Love-Gregory L, Klein S, et al. (2012) The fatty acid translocase gene CD36 and lingual lipase influence oral sensitivity to fat in obese subjects. J Lipid Res 53, 561-566.

19. Mizak I, Sery O, Plesnik J, et al. (2015) The A allele of cluster of differentiation 36 (CD36) SNP 1761667 associates with decreased lipid taste perception in obese Tunisian women. Br J Nutr 113, 1330-1337.

20. Sayed A, Sery O, Plesnik H, et al. (2015) CD36 AA genotype is associated with decreased lipid taste perception in young obese, but not lean, children. Int J Obes 39, 920-924.

21. Melis M, Sollai G, Muroni P, et al. (2015) Associations between orosensory perception of oleic acid, the common single nucleotide polymorphisms (rs1761667 and rs1527483) in the CD36 gene, and 6-n-propylthiouracil (PROP) tasting. Nutrients 7, 2068-2084.

22. Kles KA \& Chang EB (2006) Short-chain fatty acids impact on intestinal adaptation, inflammation, carcinoma, and failure. Gastroenterology 130, S100-S105.

23. Galindo MM, Voigt N, Stein J, et al. (2011) G protein-coupled receptors in human fat taste perception. Chem Senses 37, $123-139$.

24. Gilbertson T, Leonardelli M \& Wolf R (2010) Optimizing blown film line layouts for improved surface treating performance. J Plast Film Sheeting 26, 83-104.

25. Running CA \& Hayes JE (2016) Individual differences in multisensory flavor perception. In Multisensory Flavor Perception, pp. 185-210 [B Piqueras-Fiszman and C Spence, editors]. Boston, MA: Elsevier.

26. Hayes JE, Feeney EL \& Allen AL (2013) Do polymorphisms in chemosensory genes matter for human ingestive behavior? Food Qual Prefer 30, 202-216.

27. Tepper BJ \& Nurse RJ (1997) Fat perception is related to PROP taster status. Physiol Behav 61, 949-954.

28. Tepper BJ \& Nurse RJ (1998) PROP taster status is related to fat perception and preference. Ann N Y Acad Sci 855, 802-804.

29. Hayes JE \& Duffy VB (2007) Revisiting sugar-fat mixtures: sweetness and creaminess vary with phenotypic markers of oral sensation. Chem Senses 32, 225-236.
30. Drewnowski A, Henderson SA \& Barratt-Fornell A (1998) Genetic sensitivity to 6-n-propylthiouracil and sensory responses to sugar and fat mixtures. Physiol Behav 63, $771-777$.

31. Lim J, Urban L \& Green BG (2008) Measures of individual differences in taste and creaminess perception. Chem Senses 33, 493-501.

32. Heinze JM, Preissl H, Fritsche A, et al. (2015) Controversies in fat perception. Physiol Behav 152, 479-493.

33. Kamerud JK \& Delwiche JF (2007) Individual differences in perceived bitterness predict liking of sweeteners. Chem Senses 32, 803-810.

34. Keast RS \& Roper J (2007) A complex relationship among chemical concentration, detection threshold, and suprathreshold intensity of bitter compounds. Chem Senses 32, 245-253.

35. Yackinous C \& Guinard J-X (2001) Relation between PROP taster status and fat perception, touch, and olfaction. Physiol Behav 72, 427-437.

36. Miller IJ \& Reedy FE (1990) Variations in human taste bud density and taste intensity perception. Physiol Behav $\mathbf{4 7}$, 1213-1219.

37. Zuniga JR, Davis SH, Englehardt RA, et al. (1993) Taste performance on the anterior human tongue varles with fungiform taste bud density. Chem Senses 18, 449-460.

38. Zhang G, Zhang H, Wang X, et al. (2008) The relationship between fungiform papillae density and detection threshold for sucrose in the young males. Chem Senses 34, 93-99.

39. Bokor S, Legry V, Meirhaeghe A, et al. (2010) Single-nucleotide polymorphism of CD36 locus and obesity in European adolescents. Obesity 18, 1398-1403.

40. Heni M, Mussig K, Machicao F, et al. (2011) Variants in the CD36 gene locus determine whole-body adiposity, but have no independent effect on insulin sensitivity. Obesity 19, 1004-1009.

41. Ichimura A, Hirasawa A, Poulain-Godefroy O, et al. (2012) Dysfunction of lipid sensor GPR120 leads to obesity in both mouse and human. Nature $\mathbf{4 8 3}, 350$.

42. Waguri T, Goda T, Kasezawa N, et al. (2013) The combined effects of genetic variations in the GPR120 gene and dietary fat intake on obesity risk. Biomed Res 34, 69-74.

43. Love-Gregory L, Sherva R, Schappe T, et al. (2011) Common CD36 SNPs reduce protein expression and may contribute to a protective atherogenic profile. Hum Mol Genet 20, 193-201.

44. Vestmar MA, Andersson EA, Christensen CR, et al. (2016) Functional and genetic epidemiological characterisation of the FFAR4 (GPR120) p. R270H variant in the Danish population. J Med Genet 53, 616-623.

45. Heinze JM, Costanzo A, Baselier I, et al. (2018) Detection thresholds for four different fatty stimuli are associated with increased dietary intake of processed high-caloric food. Appetite 123, 7-13.

46. Asano M, Hong G, Matsuyama Y, et al. (2016) Association of oral fat sensitivity with body mass index, taste preference, and eating habits in healthy Japanese young adults. Tohoku J Exp Med 238, 93-103.

47. Martínez-Ruiz NR, Lopez-Diaz JA, Wall-Medrano A, et al. (2014) Oral fat perception is related with body mass index, preference and consumption of high-fat foods. Physiol Behav 129, 36-42.

48. Stewart JE, Seimon RV, Otto B, et al. (2011) Marked differences in gustatory and gastrointestinal sensitivity to oleic acid between lean and obese men. Am J Clin Nutr 93, 703-711.

49. Narukawa M, Kurokawa A, Kohta R, et al. (2017) Participation of the peripheral taste system in aging-dependent changes in taste sensitivity. Neuroscience 358, 249. 
50. Spielman AI, Pepino MY, Feldman R, et al. (2010) Technique to collect fungiform (taste) papillae from human tongue. J Vis Exp 42, e2201.

51. Archer NS, Liu D, Shaw J, et al. (2016) A comparison of collection techniques for gene expression analysis of human oral taste tissue. PLOS ONE 11, e0152157.

52. LifeTechnologies (2010) TRIzol Reagent. Carlsbad, CA: Life Technologies

53. Hummon AB, Lim SR, Difilippantonio MJ, et al. (2007) Isolation and solubilization of proteins after TRIzol ${ }^{\circledR}$ extraction of RNA and DNA from patient material following prolonged storage. Biotechniques $\mathbf{4 2}, 467$.

54. Department of Health and Ageing (2010) User Guide 2007 Australian National Children's Nutrition and Physical Activity Survey. Canberra: Commonwealth Government.

55. Haryono RY, Sprajcer MA \& Keast RS (2014) Measuring oral fatty acid thresholds, fat perception, fatty food liking, and papillae density in humans. $J$ Vis $\operatorname{Exp} \mathbf{8 8}, 1-12$.

56. Costanzo A, Orellana L, Nowson C, et al. (2017) Fat taste sensitivity is associated with short-term and habitual fat intake. Nutrients 9, 781

57. Simons PJ, Kummer JA, Luiken JFP, et al. (2011) Apical CD36 immunolocalization in human and porcine taste buds from circumvallate and foliate papillae. Acta Histochem 113, 839-843.

58. Ozdener MH, Subramaniam S, Sundaresan S, et al. (2014) CD36-and GPR120-mediated $\mathrm{Ca}^{2+}$ signaling in human taste bud cells mediates differential responses to fatty acids and is altered in obese mice. Gastroenterology 146, 995-1005.e5.

59. Cortés A, Mellombo M, Mgone C, et al. (2005) Adhesion of Plasmodium falciparum-infected red blood cells to CD36 under flow is enhanced by the cerebral malaria-protective trait South-East Asian ovalocytosis. Mol Biochem Parasitol 142 , $252-257$.

60. Laugerette F, Passilly-Degrace P, Patris B, et al. (2005) CD36 involvement in orosensory detection of dietary lipids, spontaneous fat preference, and digestive secretions. J Clin Invest 115, 3177.

61. Itoh Y, Kawamata Y, Harada M, et al. (2003) Free fatty acids regulate insulin secretion from pancreatic $\beta$ cells through GPR40. Nature 422, 173-176.
62. Cartoni C, Yasumatsu K, Ohkuri T, et al. (2010) Taste preference for fatty acids is mediated by GPR40 and GPR120. J Neurosci 30, 8376-8382.

63. Yousefi S, Cooper PR, Potter SL, et al. (2001) Cloning and expression analysis of a novel G-protein-coupled receptor selectively expressed on granulocytes. I Leukoc Biol 69, 1045-1052.

64. Inada $\mathrm{H}$, Kawabata F, Ishimaru $\mathrm{Y}$, et al. (2008) Off-response property of an acid-activated cation channel complex PKD1L3-PKD2L1. EMBO Rep 9, 690-697.

65. Ishii S, Misaka T, Kishi M, et al. (2009) Acetic acid activates PKD1L3-PKD2L1 channel - a candidate sour taste receptor. Biochem Biophys Res Commun 385, 346-350.

66. Pluznick JL (2014) 'Extra' sensory perception: the role of Gpr receptors in the kidney. Curr Opin Nephrol Hypertens 23, 507.

67. Nilsson NE, Kotarsky K, Owman C, et al. (2003) Identification of a free fatty acid receptor, FFA2R, expressed on leukocytes and activated by short-chain fatty acids. Biochem Biophys Res Commun 303, 1047-1052.

68. Senga T, Iwamoto S, Yoshida T, et al. (2003) LSSIG is a novel murine leukocyte-specific GPCR that is induced by the activation of STAT3. Blood 101, 1185-1187.

69. Le Poul E, Loison C, Struyf S, et al. (2003) Functional characterization of human receptors for short chain fatty acids and their role in polymorphonuclear cell activation. $\mathrm{J} \mathrm{Biol} \mathrm{Chem}$ 278, 25481-25489.

70. Drewnowski A (1985) Food perceptions and preferences of obese adults: a multidimensional approach. Int J Obes $\mathbf{9}$, 201-212.

71. Risso DS, Giuliani C, Antinucci M, et al. (2017) A bio-cultural approach to the study of food choice: the contribution of taste genetics, population and culture. Appetite 114, 240.

72. Abdoul-Azize S, Selvakumar S, Sadou H, et al. (2014) $\mathrm{Ca}^{2+}$ signaling in taste bud cells and spontaneous preference for fat: unresolved roles of CD36 and GPR120. Biochimie 96, 8-13.

73. Gilbertson TA \& Khan NA (2014) Cell signaling mechanisms of oro-gustatory detection of dietary fat: advances and challenges. Progr Lipid Res 53, 82-92. 